\title{
Plasmakinetic vaporization versus plasmakinetic resection to treat benign prostatic hyperplasia: A prospective randomized trial with 1 year follow-up
}

\author{
Mert Ali Karadag, MD; ${ }^{*}$ Kursat Cecen, MD; ${ }^{*}$ Aslan Demir, MD; ${ }^{*}$ Ramazan Kocaaslan, MD; ${ }^{*}$ Fatih Altunrende, $M D^{\dagger}$ \\ *Faculty of Medicine, Department of Urology, Kafkas University, Kars, Turkey; †Department of Urology, Istanbul Bilim University, Beşiktaş/istanbul, Turkey
}

Cite as: Can Urol Assoc J 2014;8(9-10):e595-9. http://dx.doi.org/10.5489/cuaj.1902 Published online September 9, 2014.

\section{Abstract}

Introduction: We evaluate the efficacy and outcomes of plasmakinetic vaporization (PKVP) and plasmakinetic resection (PKR) to treat benign prostatic hyperplasia (BPH).

Methods: A total of 183 patients with BPH underwent plasmakinetic prostatic surgery between 2008 and 2012 at Kars State Hospital and Kafkas University Faculty of Medicine, Turkey. After clinical and preoperative evaluation, the patients were randomized to PKRP or PKVP groups sequentially by using computer-generated numbers. Group 1 included 96 patients treated with PKR. Group 2 included 87 patients treated with PKVP. Patients in both groups were compared in terms of hemoglobin drop, operation time, catheter duration, reobstruction, incontinence and recatheterization. Results: When we compared the maximum flow rates (Qmax values) at the 12th month, there was no statistical difference between 2 groups. Group 1 had a mean Qmax value of $17.92 \pm 3.819$ and Group 2 had a $18.15 \pm 3.832$ value $(p>0.05)$. There was a statistical difference between the groups in terms of hemoglobin drop, catheter duration and operation time. The mean catheter duration in Group 1 was $3.74 \pm 1.049$ days, and in Group 2 it was $2.64 \pm 0.849$ days $(p<0.05)$. Operation time was statistically longer in Group 2 (PKVP) and hemoglobin drop was statistically higher in Group 1 (PKR).

Conclusion: PKVP for BPH is safe and effective. When compared with PKRP, it provides a significantly shorter catheter duration and less bleeding due to hemostasis control with similar IPSS and Qmax improvements after 1 year.

\section{Introduction}

Bipolar electrusurgical technology, like plasmakinetic resection (PKR) and plasmakinetic vaporization (PKVP) of the prostate, has been introduced in the market for decreasing complications associated with transurethral resection of the prostate (TURP). ${ }^{1-2}$ Many studies have compared PKR and PKVP with TURP and they concluded that plasmakinetic technology had similar outcomes and less morbidity in the early and late follow-up periods. ${ }^{1-4}$ However, in one study comparing long-term results of transurethral vaporization of the prostate using plasmakinetic energy with conventional TURP, the authors concluded that the outcomes, like the International Prostate Symptom Score (IPSS) and maximum flow rates (Qmax), of PKVP after 3 years was statistically worse than TURP $(p<0.05) .^{5}$

Most studies until now have compared the outcomes of PKR or PKVP in BPH with monopolar TURP..$^{1-3,5-6}$ In this study, we compared the efficacy and outcomes of PKVP with PKR in the treatment of $\mathrm{BPH}$.

\section{Methods}

Our study was approved by the local ethics committee at Kafkas University and performed in accordance with the World Medical Association Helsinki Declaration. Every patient understood and signed consent forms.

We performed a routine physical examinations, including digital rectal examination (DRE) with complete blood count, urine analysis, uroflowmetry, postvoid residual urine (PVR), serum creatinine, ultrasonography of urinary system (USG) and prostate-specific antigen (PSA) measurement in all patients who were admitted to our outpatient clinic for lower urinary tract symptoms (LUTS). The prostatic volume and PVR were measured with transabdominal USG in all cases. The IPSS of each patient was compiled. Uroflowmetry was performed with a minimum accepted urinary volume of $150 \mathrm{~mL}$.

Patients were included in the study if they had moderate to severe LUTS, based on their IPSS, requiring surgery, recurrent urinary retention, failed medical therapy (at least 21 days) and obstructive pressure flow study or Qmax less than $10 \mathrm{~mL} / \mathrm{s}$. Patients were excluded if there was suspicion of prostatic adenocarcinoma, if they had an abnormal 
Karadag et al.

DRE or elevated PSA, known urethral stricture or neurogenic bladder and a history of prostate surgery.

A total of 183 patients diagnosed with $\mathrm{BPH}$ underwent plasmakinetic prostatic surgey between 2008 and 2012 at the Kars State Hospital and Kafkas University Faculty of Medicine. The average patient age was 67.32 years. After clinical and preoperative evaluation, patients were randomized to the PKR (Group 1) or Group 2 (PKVP) sequentially by using computer-generated numbers.

Group 1 included 96 patients with a mean age of $66.72 \pm 6.04$. The mean prostate volume and PVR of Group 1 was $50.6 \pm 16.9 \mathrm{~mL}$ and $96.03 \pm 103.05 \mathrm{~mL}$, respectively. Group 2 included 87 patients with a mean age of $67.98 \pm 6.069$. The mean prostate volume and PVR of Group 2 was $50.9 \pm 16.5 \mathrm{~mL}$ and $82.83 \pm 74.43 \mathrm{~mL}$, respectively. All patients in both groups were compared in terms of hemoglobin drop, operation time, catheter duration, reobstruction, incontinence and recatheterization. Hemoglobin drop was calculated in blood samples on postoperative day 1. Success was evaluated with IPSS and Qmax value at postoperative month 12. Cases with an IPSS $<14$ and Qmax $>15 \mathrm{~mL} / \mathrm{s}$ were considered successful.

The data were analyzed by using SPSS, Windows v. 6 . The mean, median and standard deviation values were used for descriptive statistics. The characteristics with normal and non-normal distributions were compared by using Student t-test and the Mann-Whitney tests, respectively. A $p$ value of $<0.05$ was considered significant. Depending on the results of a pilot study which was perfomed in our department and the results of Muslumanoglu's and Hon's study ${ }^{1-2}$ dealing with the operation time and hemoglobin drop after PKVP, we calculated the sufficient sample size for our study with a power of 80, upon consultation with a biostatistician. Although our sample size was much higher than calculated, it was due to the routine application of these 2 procedures in our department for patients with $\mathrm{BPH}$.

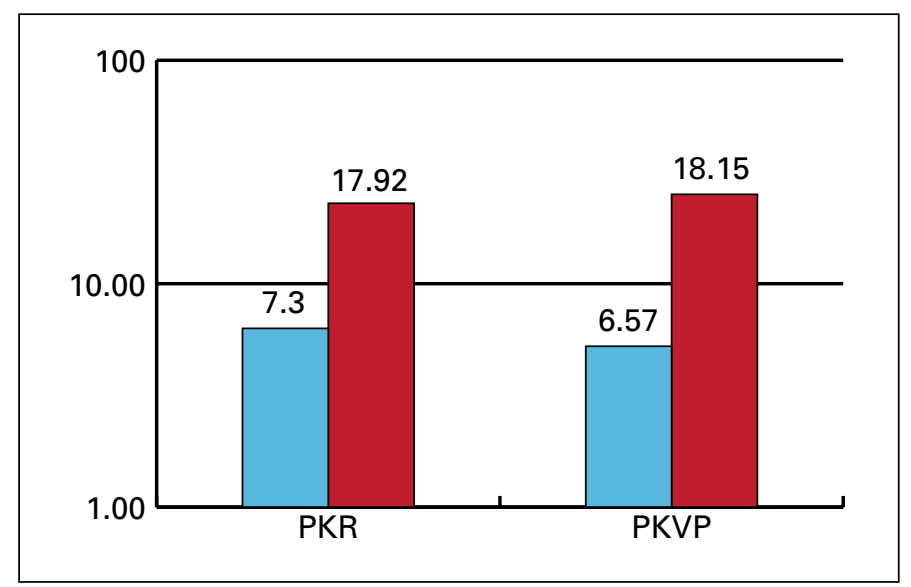

Fig. 1. Qmax (maximum flow rates) improvements after 1 year. PKR: plasmakinetic resection; PKVP: plasmakinetic vaporization.
All of the procedures were performed by 1 experienced urologist. All patients were operated under spinal anesthesia in the lithotomy position. Cephazolin sodium $1 \mathrm{~g}$ intravenously was administered for preoperative antibiotic prophylaxis. A SuperPulse (Olympus Inc.) generator was used and PKR was performed with a 27F continuous flow resectoscope and PKVP was maintained with a plasmakinetic loop electrode of same system (Gyrus Medical Ltd, UK) used for removing the prostatic tissues by vaporization. Irrigation fluid during the surgery was isotonic saline for both groups.

\section{Results}

Data for all patients were obtained at the end of 12 months. There were no statistically significant differences between both groups in terms of age, prostatic volume, PVR, preoperative Qmax value and IPSS ( $p>0.05)$. The mean preoperative Qmax value of Group 1 and Group 2 was $7.30 \pm 2.59 \mathrm{~mL} / \mathrm{s}$ and $6.57 \pm 2.731 \mathrm{~mL} / \mathrm{s}(p=0.06)$, respectively. The IPSS of Group 1 and Group 2 was $20.52 \pm 5.71$ and $21.46 \pm 5.68$ $(p=0.26)$. The mean PVR of Group 1 and Group 2 decreased to $34.38 \pm 26.01 \mathrm{~mL}$ and $40.14 \pm 27.54 \mathrm{~mL}$, respectively, at postoperative month 1. The mean Qmax value of Group 1 on the first month increased to $16.7 \pm 3.73 \mathrm{~mL} / \mathrm{s}$ and the IPSS decreased to $11.9 \pm 3.82$. Group 2 had a mean Qmax value of $17.01 \pm 3.65 \mathrm{~mL} / \mathrm{s}$ and an IPSS of $11.7 \pm 3.71$ at postoperative month 1 . The difference between the groups was not statistically significant, but the improvements in baseline values for both groups were statistically significant $(p<0.05)$.

When we compared the Qmax values at month 12, there was no statistical difference between both groups. Group 1 had a mean Qmax value of $17.92 \pm 3.819$ and Group 2 with $18.15 \pm 3.832(p=0.69)$. We also did not find any significant differences in IPSS between PKR and PKVP groups at month 12. The mean IPSS in Group 1 and Group 2 were

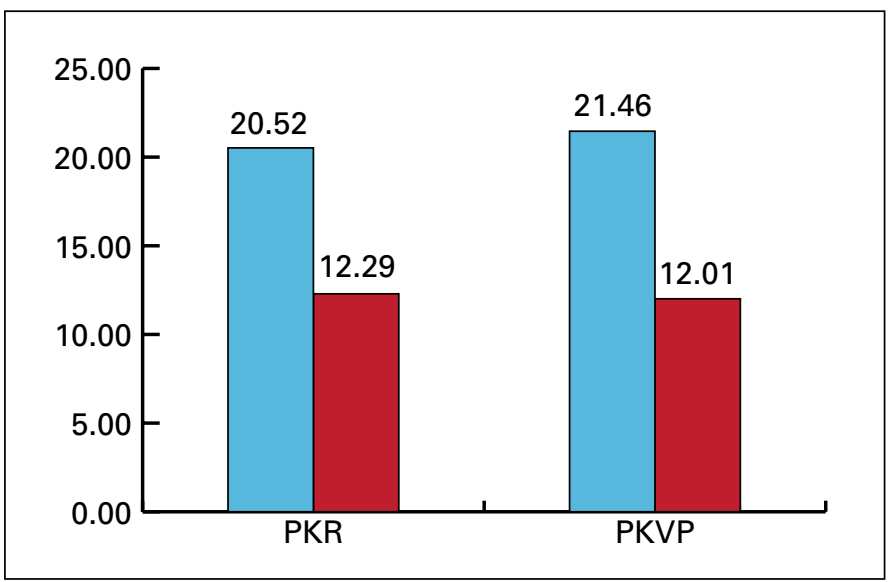

Fig. 2. IPSS improvements after 1 year. IPSS: International Prostate Symptom Score; PKR: plasmakinetic resection; PKVP: plasmakinetic vaporization. 
$12.29 \pm 3.758$ and $12.01 \pm 3.677$, respectively $(p=0.61)$. The overall IPSS and Qmax values significantly improved from the baseline values at the end of first year (Fig. 1, Fig. 2).

There was a statistical difference between both groups in terms of hemoglobin drop, catheter duration and operation time. The mean catheter duration of Group 1 was $3.74 \pm 1.04$ days, and in Group 2 it was $2.64 \pm 0.849$ days $(p<0.001)$. The mean operation time of Group 1 and Group 2 was $52.07 \pm 8.682$ minutes and $61.08 \pm 10.256$ minutes, respectively $(p<0.001)$. Group 1 (PKR) had a mean hemoglobin drop value of $1.28 \pm 0.75 \mathrm{~g} / \mathrm{dL}$, on the other hand this value was only $0.55 \pm 0.62 \mathrm{~g} / \mathrm{dL}$ in Group 2 (PKVP) $(p<0.001)$ (Table 1).

Only in 2 cases from Group 1 (PKR) did we crossover to PKVP due to inadequate hemostatis control. We stopped all procedures and convereted to PKVP for the final part of the operations. After conversion, we obtained successful hemostasis control. We did not switch between the operations due to incomplete tissue removal. There were no significant differences between both groups in terms of infravesical obstruction, incontinence and recatheterization. Infravesical obstruction was observed in 4 patients from Group 1 and 3 patients from Group $2(p>0.05)$. These patients required re-treatment and operated with the same modalities at an average of 12 months after initial bipolar procedures. Every patient satisfactorily voided after the second plasmakinetic procedure. Early incontinence (mostly urgency type) was observed in 3 patients from Group 1 and 1 patient from Group 2; this incontinence disappeared for these 4 patients at the end of the third month. Eleven patients in Group 2 and 6 patients in Group 1 required urethral catheterization $(0.13$ vs. 0.06) ( $p=0.138)$. We did not encounter any massive hematuria or urinary tract infections. Patients admitted to the emergency department needed recatheterization; most of these patients were the ones treated with PKVP (Group 2).

\section{Discussion}

There are some disadvantages and complications of monopolar TURP, such as bleeding, TUR syndrome, longer learning curve and prolonged catheterization. ${ }^{7}$ In a prospective multicentre trial involving the data of 10654 patients, morbidity of TURP was reported as high as $11.1 \%$, with a mortality rate of $0.1 \%{ }^{8}$ Many investigations compared the outcomes and safety of plasmakinetic technology with monopolar TURP. ${ }^{1-3,6}$ They concluded that plasmakinetic surgery maintained similar results with monopolar TURP with less morbidity. In our study, the improvements in Qmax and IPSS of PKR and PKVP groups over baseline values at the end month 12 were statistically significant. Moreover, there was no statistically significant success difference between both groups. This was due to the preferred same energy source. After introducing plasmakinetic technology in our department, most patients with BPH treated with this system had acceptable outcomes.

The mechanism of plasmakinetic depends on a plasma formation that creates an electrically conductive cloud, when radiofrequency energy contacts tissue. ${ }^{9}$ An advantage of plasmakineti is cutting the tissues at much lower average temparature (as low as $50^{\circ} \mathrm{C}$ ) than conventional electrocautery. ${ }^{10}$ In the PKVP procedure, the current between the active and return electrode forms a plasma ball, which vaporizes the tissue on contact with a very high temperature and low thermal mass. ${ }^{11}$ Similar to PKR, thermal damage is less than $1 \mathrm{~mm}$. Another advantage of PKVP is maintaining a maximal hemostasis, when operated under low voltage (80-100 V). ${ }^{4}$ Coagulation mode can be used at the end of the procedure for obtaining maximum hemostasis. We also performed hemostasis under coagulation mode at the end of PKVP procedures. In our study, hemoglobin drop rate of PKVP group was significantly lower than in the PKR group and this may be attributed to coagulation advantage of vaporization mode. Also vaporization of the tissue and simultaneous coagulation mode provide a very clear vision in the operating field and eliminates the risk of TUR syndrome.

In one study, comparing the results of transurethral vaporization with plasmakinetic and monopolar TURP during the 1-year follow-up, the group concluded that less irrigation requirement after PKVP at the postoperative period was the main reason for shorter hospitalization. ${ }^{11}$ The same study also reported a shorter operation time with PKVP compared with TURP. The mean operation time of TURP was $55 \pm 11.1$ minutes; with PKVP, it was $40.3 \pm 15$ minutes and this difference between TURP and PKVP was statistically significant. Muslumanoglu and colleagues compared the operation time between PKR and TURP. ${ }^{1}$ They revealed that the mean operating time of PKR and TURP was $40.3 \pm 11.5$ minutes and $57.8 \pm 13.4$ minutes, respectively $(p<0.001)$. In our study, the mean operation time of PKR and PKVP was $52.07 \pm 8.682$ minutes and $61.08 \pm 10.256$ minutes, respectively $(p<0.05)$. The longer operating time of PKVP might be attributed to the hemostasis motivation of

\begin{tabular}{|c|c|c|c|c|c|c|c|c|}
\hline & $\mathbf{n}$ & age & Bleeding (g/dL) & Op. Time (min.) & Catheter duration & Obstruction (n) & Recatheterization (n) & Incontinence (n) \\
\hline PKRP & 96 & $66.72 \pm 6.04$ & $1.28 \pm 0.75$ & $52.07 \pm 8.682$ & $3.74 \pm 1.04$ & 4 & 6 & 3 \\
\hline PKVP & 87 & $67.98 \pm 6.069$ & $0.55 \pm 0.62$ & $61.08 \pm 10.256$ & $2.64 \pm 8.849$ & 3 & 11 & 1 \\
\hline
\end{tabular}

PKR: PlasmaKinetic resection; PKVP: PlasmaKinetic vaporization. 
the surgeon at the end of the procedure. Coagulation mode was used for hemostasis after vaporization of adenomatous tissue.

Karaman and colleagues compared the irrigation time of patients who underwent either TURP or PKVP. ${ }^{11}$ The irrigation time of the PKVP group was statistically lower than in the TURP group ( $6 \pm 3 \mathrm{hr}$ vs. $20 \pm 8 \mathrm{hr}$ ). This difference may be attributed to less bleeding and coagulation - both advantages of PKVP. The same advantages were also seen in PKR. ${ }^{1}$ Less intraoperative and postoperative irrigation were required in PKR patients compared with conventional TURP. In our study, we did not compare the groups in terms of irrigated fluid or irrigation time. This same group from the Karaman study also revealed a mean operation time of $40.3 \pm 15$ minutes for the patients who underwent PKVP. ${ }^{11}$ In our study, the mean operation time of the PKVP group (Group 2) was $61.08 \pm 10.256$ minutes. Although the mean prostate volume of the Karaman study and our current study was similar, this difference in operation time might be explained by surgeon experience and the motivation for maximal hemostasis.

Hon and colleagues also compared the serum hemoglobin change between patients who underwent TURP and PKVP. ${ }^{2}$ They concluded that mean hemoglobin drop in the PKVP group was $0.8 \pm 0.9 \mathrm{~g} / \mathrm{dL}$ and this was statistically lower than in the TURP group. We also performed a hemoglobin check between both groups on postoperative day 1 . The hemoglobin drop was statistically higher in the PKR group compared to the PKVP group $(1.28 \mathrm{~g} / \mathrm{dL}$ vs. $0.55 \mathrm{~g} / \mathrm{dL})$. We think that the coagulation advantage of PKVP created this difference, even though both procedures used the same energy source. In our study, the average hemoglobin decline in the PKVP group is similar to the results seen in the Hon study. ${ }^{2}$ In a very recent randomized prospective trial comparing the outcomes of thulium laser enucleation and PKR, the authors reported a mean operation time of 47.4 minutes and a mean hemoglobin drop of $0.30 \mathrm{~g} / \mathrm{dL}$ in patients who underwent PKR. ${ }^{12}$ In our study in the PKR group, the mean hemoglobin drop and the mean operation time was $1.28 \pm 0.75 \mathrm{~g} / \mathrm{dL}$ and $52.07 \pm 8.682$ minutes. There was a similarity between the results of our study and the results by Kang and colleagues ${ }^{12}$ in terms of operation time, but the mean hemoglobin drop in patients who underwent PKR in our study was higher than the drop in the other study. This study's hemoglobin drop was similar to our PKVP group. A similar study from China compared thulium laser and PKR; the study revealed a mean operation time of $30.14 \pm 15.9$ minutes in 50 patients in the PKR group. ${ }^{13}$ The mean operation time of this group was much lower than in our study. This difference might be attributed to the experience of the other department. Huang and colleagues compared the coagulation depth and bleeding of PKR with monopolar TURP. ${ }^{14}$ The authors obtained a mean hemoglobin drop of $0.71 \pm 0.42 \mathrm{~g} / \mathrm{dL}$ after PKR. This result was similar with our study's mean hemoglobin drop.

Sinanoglu and colleagues compared the postoperative outcomes of PKR and monopolar TURP in patients with comorbidities. ${ }^{15}$ The authors noted a mean operation time $60.3 \pm 23.8$ minutes for PKR. Although the prostate sizes were similar with our study, this difference might be attributed to different patient characteristics, like comorbidities. In the Sinanoglu study, the mean IPSS decreased to $9 \pm 7.9$ and the mean Qmax increased to $18.8 \pm 6.4 \mathrm{~mL} / \mathrm{s}$ at the end of 12 months. Our mean postoperative Qmax value of $17.92 \pm 3.819$ was similar, but the mean IPSS value at 12 months was $12.29 \pm 3.758$. The IPSS in our study was higher. We should mention one key point to explain the moderate IPSS which is discordant with high Qmax of the same patients who underwent either PKR or PKVP in our study. The main reason is the low education level of these patients. It was difficult for most patients to complete the IPSS questionnaire. This is also an explanation where there was discordance between IPSS and uroflowmetry results. IPSS is a subjective parameter which depends on patient's answers and the uroflowmetry is an objective parameter.

PKVP can be safely performed as an outpatient procedure. ${ }^{4}$ Eaton and colleagues found that patients who underwent PKVP might be discharged on the same day without a catheter. In our study, the mean catheter duration of Group 1 (PKR) was 3.74 days and for Group 2 it was 2.64 days $(p<0.05)$. The average duration of the urethral catheter in the Karaman study was 35 hours. ${ }^{11}$ This difference may be attributed to experience and the fact that most of our patients were farmers and coming to our centre from peripheral localizations or villages (likely $80-90 \mathrm{~km}$ away). To avoid surprises, such as early globe or massive hematuria, we preferred a longer average catheter time for patients' safety and comfort.

In our study, 11 patients treated with PKVP required recatheterization after removal of the initial catheter. Only 6 patients in Group 1 were recatheterized in the early period. Of the 11 patients in the PKVP group, we performed diagnostic cystoscopy in 4 patients due to difficulty in applying urethral catheter. In all patients, we observed severe edema around the verumontonum and apex of the prostate that obstructed the urethra. We had recatheterized these patients for 1 week with antiinflammatory therapy and the catheters were removed after 1 week. All of the patients voided satisfactorily after being free of catheter. In our series, we did not encounter any urinary tract infection or massive hematuria. We prescribed antibiotics to patients who were disharged and advised them to complete the medicine duration. We did not encounter massive hematuria due to the hemostasis motivation of the surgeon. Compliance and hemostasis motivation of the surgeon mainly depended on the aforementioned socioeconomic factors of our patient population.

The pathologic evaluation of Group 1 did not reveal any 
unsuspected prostatic carcinoma. Pathologic examination after PKVP could not be maintained due to absence of tissue sampling. This can be considered as a disadvantage of PKVP. By using resection loops like Plasma-sect, this problem might be overcome.

There are several limitations in our study. First of all, our study included a small sample size. There are 2 institutions involving in this study, but the procedures were performed by only 1 experienced surgeon. Due to the variations in surgical skills between surgeons, it is difficult to establish standardizations regarding surgical interventions.

\section{Conclusion}

PKVP to manage BPH is safe and effective. When compared with $\mathrm{PKR}$, it provides a significantly shorter catheter duration and less bleeding due to advantage of hemostasis control with similar IPSS and Qmax improvements after 1 year.

Competıng interests: Dr. Karadag, Dr. Cecen, Dr. Demir, Dr. Kocauslan and Dr. Altunrende all declare no competing financial or personal interests.

This paper has been peer-reviewed.

\section{References}

1. Muslumanoglu AY, Yuruk E, Binbay M, et al. Transurethral resection of prostate with plasmakinetic energy: 100 months results of a prospective randomized trial. BJU Int 2011;110:546-9. http://dx.doi. org/10.1111/i.1464-410X.2011.10770.x

2. Hon NHY, Brathwaite D, Hussain Z, et al. A prospective, randomized trial comparing conventional transurethral prostate resection with PlasmaKinetic ${ }^{\text {TM }}$ Vaporization of the prostate: Physiological changes, early complications and long-term follow up. J Urol 2006;176:205-9. http://dx.doi.org/10.1016/ S0022-5347(06)00492-7
3. Botto $\mathrm{H}$, Lebret $\mathrm{T}$, Barre $\mathrm{P}$, et al. Electrovaporization of the prostate with the Gyrus device. J Endourol 2001;15:313-6. http://dx.doi.org/10.1089/089277901750161917

4. Eaton $\mathrm{AC}$, Francis RN. The provision of transurethral prostatectomy on a day case basis using bipolar plasmakinetic technology. BJU Int 2002;89:354-7. http://dx.doi.org/10.1046/j.1464-410X.2002.02673.x

5. Kaya $C$, Ilktac $A$, Gokmen $E$, et al. The long-term results of transurethral vaporization of the prostate using plasmakinetic energy. BJU Int 2007;99:845-8. http://dx.doi.org/10.1111/i.1464410X.2006.06683.x

6. Tefekli A, Muslumanoglu AY, Baykal M, et al. A hybrid technique using bipolar energy in transurethral prostate surgery: A prospective, randomized comparison. J Urol 2005;174:1339-43. http://dx.doi. org/10.1097/01.ju.0000173075.62504.73

7. Metbust WK, Holtgrewe HL, Cockett AT, et al. Transurethral prostatectomy: immediate and postoperative complications. A comparative study 13 participating institutions evaluating 3885 patients. J Urol 1989;141:243-7.

8. Reich 0, Gratzke C, Bachmann A, et al. Morbidity, mortality and early outcome of transurethral resection of the prostate: A prospective multicenter evaluation of 10654 patients J Urol 2008; 180:246-9. http:// dx.doi.org/10.1016/i.juro.2008.03.058

9. Loh SA, Carlson GA, Chang El, et al. Comparative healing of surgical incisions created by PEAK Plasmo Blade, conventional electrosurgery, and a scalpel. Plas Reconstr Surg 2009;124:1849-59. http://dx.doi. org/10.1097/PRS.0b013e3181bcee87

10. Ruidiaz ME, Messmer D, Atmodjo DY, et al. Comparative healing of human cutaneous surgical incisions created by the PEAK PlasmaBlade, conventional electrosurgery, and a standart scalpel. Plas Reconstr Surg 2011;128:104-11. http://dx.doi.org/10.1097/PRS.0b013e3182174led

11. Karaman MI, Kaya C, Ozturk M, et al. Comparison of transurethral vaporization using PlasmaKinetic ${ }^{\top M}$ energy and transurethral resection of prostate: Randomized trial with 1 year follow-up. J Endourol 2005;19:734-7. http://dx.doi.org/10.1089/end.2005.19.734

12. Yang $Z$, Wang $X$, Liu T. Thulium laser enucleation versus plasmakinetic resection of the prostate: $A$ randomized prospective trial with18-month follow up. Urology 2013;81:396-400. http://dx.doi. org/10.1016/i.urology.2012.08.069

13. Peng $B$, Wang $G C$, Zheng JH, et al. A comparative study of thulium laser resection of the prostate and bipolar transurethral Plasmakinetic prostatectomy for treating benign prostatic hyperplasia. BJU Int 2013;111:633-7. http://dx.doi.org/10.1111/i.1464-410X.2012.11610.x

14. Huang $X$, Wang L, Wang $X$, et al. Bipolar transurethral resection of the prostate causes deeper coagulation depth and less bleeding than monopolar transurethral prostatectomy. Urology 2012;80:1116-20. http://dx.doi.org/10.1016/j.urology.2012.07.024

15. Sinanoglu 0 , Ekici S, Tatar $M N$, et al. Postoperative outcomes of Plasmakinetic transurethral resection of the prostate compared to monopolar transurethral resection of the prostate in patients with comorbidities. Urology 2012;80:402-6. htrp://dx.doi.org/10.1016/j.urology.2012.02.029

Correspondence: Dr. Mert Ali Karadag, Faculty of Medicine, Department of Urology, Kafkas University, Kars, Turkey; karadagmert@yahoo.com 